Sonderdruck aus
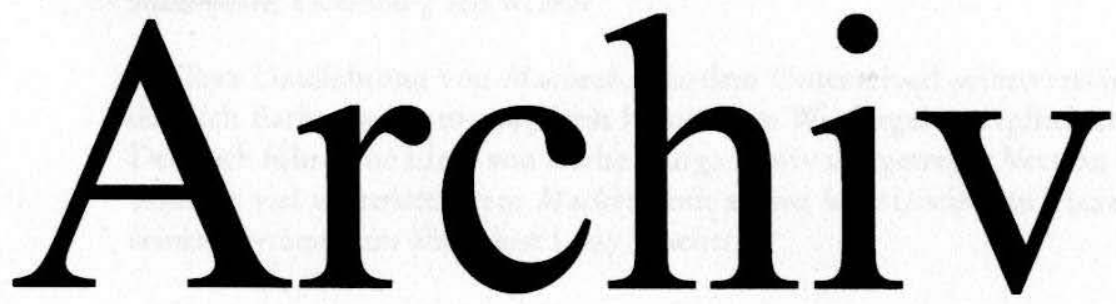

für das Studium der neueren Sprachen und Literaturen

Herausgegeben von

HORST BRUNNER

CHRISTA JANSOHN

MANFRED LENTZEN

DIETER MEHL

243. Band

158. Jahrgang

1. Halbjahresband 2006 


\section{Kleinere Beiträge}

\section{Cato und Facetus im Hausbuch Michaels de Leone. Zum hand- schriftlichen Nach-, Neben- und Ineinander von Latein und Deutsch im 14. Jahrhundert}

Über den Inhalt des zerstörten ersten Bandes des Hausbuchs des Würzburger Protonotars Michael de Leone ( $† 1355)$ informiert nur noch ein Register, das dem heute in der Münchener Universitätsbibliothek aufbewahrten zweiten Band vorangestellt ist (München, UB, $2^{\circ}$ Cod. ms. $\left.731,1 \mathrm{va}-2 \mathrm{rb}\right){ }^{1}$ Als Repertorium (1v) wie Capitolorum Registrum (2r) bezeichnet, weist es in den letzten vier Zeilen von $\mathrm{Bl}$. 1vb als neuntes und zehntes Stück des ersten Bandes aus: DEr katho zu latin. vnd zú tútsche. I Der facetus. Cum nichil utilius. zu latin. / vnd in / tútsch. Die Identifizierung der genannten Werke steht außer Frage: zum einen handelt es sich um die spätantiken 'Disticha Catonis', zum anderen um den hochmittelalterlichen 'Facetus Cum nibil utilius', denen beiden eine deutsche Übertragung an die Seite gestellt wurde. Auf den ersten Blick scheint die ganze Textpartie daher höchstens insofern Beachtung zu verdienen, als mit dem Facetus eines jener zwei Werke vorliegt, von denen wenigstens noch Bruchstücke erhalten sind (München, BSB, Cgm 195', 5rv - das zweite Werk ist Hugos von Trimberg 'Renner', vgl.

${ }^{1}$ Die Handschrift beschreiben ausführlich: Gisela Kornrumpf, Paul Gerhard Völker: Die deutschen mittelalterlichen Handschriften der Universitätsbibliothek München. Wiesbaden 1968 (Die Handschriften der Universitätsbibliothek München 1), S. 66-107 und S. 349. In Schwarzweiß-Abbildungen ist sie zugänglich über: Das Hausbuch des Michael de Leone (Würzburger Liederhandschrift) der Universitätsbibliothek München $\left(2{ }^{\circ}\right.$ Cod. Ms. 731), hrsg. von Horst Brunner. Göppingen 1983 (Litterae 100). Vom Liederteil des Hausbuchs, der in der Lyrikforschung unter der Sigle E firmiert, liegt überdies ein Farbfaksimile vor: Die Lieder Reinmars und Walthers von der Vogelweide aus der Würzburger Handschrift $2^{\circ}$ Cod. Ms. 731 der Universitätsbibliothek München. Bd. 1: Faksimile. Mit einer Einführung von Gisela Kornrumpf. Wiesbaden 1972. Über Michael de Leone informiert zusammenfassend Gisela Kornrumpf: Michael de Leone. In: ${ }^{2}$ VL 6 (1987), Sp. 491-503, über sein Hausbuch ausführlicher Peter Keyser: Michael de Leone († 1355) und seine literarische Sammlung. Würzburg 1966 (Veröffentlichungen der Gesellschaft für fränkische Geschichte. Reihe 9: Darstellungen aus der fränkischen Geschichte 21). Vgl. auch Ursula Peters: Literatur in der Stadt. Studien zu den sozialen Voraussetzungen und kulturellen Organisationsformen städtischer Literatur im 13. und 14. Jahrhundert. Tübingen 1983 (Studien und Texte zur Sozialgeschichte der Literatur 7), S. 138-168. Die Schreibsprache der Handschrift hat jüngst Norbert Richard Wolf präziser bestimmt: Zur Schreibsprache des Hausbuchs Michaels de Leone. In: Dorothea Klein, Elisabeth Lienert, Johannes Rettelbach (Hrsg.): Vom Mittelalter zur Neuzeit. Festschrift für Horst Brunner. Wiesbaden 2000, S. 359-368.

${ }^{2}$ Disticha Catonis, hrsg. von Marcus Boas. Amsterdam 1952; vom 'Facetus Cum nibil utilius' liegt keine kritische Ausgabe vor, so dass einstweilen zu benutzen ist: Leopold Zatočil: Cato a Facetus. Pojednáni a texty. Zu den deutschen Cato- und Facetusbearbeitungen. Untersuchungen und Texte. Brno 1952 (Spisy masarykovy university v Brně filosofická fakulta / Opera Universitatis Masarykianae Brunensis Facultas Philosophica 48), S. 287-293. 
München, BSB, Cgm 195', 1r-4v, und Nürnberg, GNM, Hs. 9030). ${ }^{3}$ Im übrigen aber handelt es sich bei den lateinischen 'Disticha Catonis' wie beim 'Facetus Cum nibil utilius' um Lektüre des Trivialunterrichts, also um Werke von auch ohne das Zeugnis des Hausbuchs beträchtlicher, dabei über den engeren Schulzusammenhang auch hinausreichender Verbreitung, ist weiterhin die im Hausbuch belegte Verbindung beider Werke mit deutschen Entsprechungen zumal in spätmittelalterlichen Schulhandschriften, wiederum ohne auf diesen Textgebrauchsraum beschränkt zu sein, recht häufig anzutreffen und erscheint die kleine Textpartie schließlich nicht einmal hinsichtlich der unmittelbaren Aufeinanderfolge gleich zwei zweisprachiger "Schultexte" sonderlich bemerkenswert. Der lateinische Facetus gibt sich nämlich schon in seinen Eingangsversen (V.1-4) selbst als eine Fortsetzung des Cato aus, so dass sich sein Anschluss ebenso in ein- wie in zweisprachigen Verwendungskontexten auch außerhalb von $\mathrm{E}$ häufig nachweisen lässt. ${ }^{4}$

Aber auf den zweiten Blick verdient das kleine Textensemble denn doch genauere $\mathrm{Be}-$ achtung, und zwar angesichts des Zeitpunkts seiner Zusammenstellung bereits in der Mitte des 14. Jahrhunderts. Denn was die spätere Überlieferung des deutschen Cato und des deutschen Facetus als einigermaßen eingespielten Standard vor Augen führt, muss deshalb im zeitlichen Umfeld des Hausbuchs noch nicht selbstverständlich gewesen sein. Nachstehend wird zu zeigen sein, dass die Cato-Facetus-Partie des Hausbuchs ein wichtiges Schlaglicht auf die Herausbildung solcher Standards im Bereich des Layouts zweisprachig dargebotener Schultexte wirft - und damit letztlich auf gewichtige Veränderungen im institutionalisierten Trivialunterricht des 14. Jahrhunderts.

${ }^{3}$ Beschreibungen der Fragmente bei Erich Petzet: Die deutschen Pergament-Handschriften Nr. 1-200 der Staatsbibliothek in München. München 1920 (Catalogus codicum manu scriptorum bibliothecae Monacensis 5,1), S. 354f., bzw. bei Lotte Kurras: Die deutschen mittelalterlichen Handschriften. Teil 1: Die literarischen und religiösen Handschriften. Anhang: Die Hardenbergschen Fragmente. Wiesbaden 1974 (Kataloge des Germanischen Nationalmuseums Nürnberg: Die Handschriften des Germanischen Nationalmuseums Nürnberg 1), S. 63; Farbabbildungen aller Bruchstücke: Horst Brunner, Hans-Günter Schmidt (Hrsg.): Vom Großen Löwenhof zur Universität. Würzburg und die deutsche Literatur im Spätmittelalter. Wiesbaden 2002, S. 23-35. Den Text des Facetus hat bereits Gustav Ehrismann bekannt gemacht und zudem zwei weitere Textzeugen dieser Übersetzungsfassung benannt: Bruchstücke eines Facetus. Germania 30 (1885), S. 284-287; Carl Schröder hat diesen drei Handschriften die Siglen G, $g^{1}$ und $\mathrm{g}^{2}$ zugewiesen und den Text nach der vollständigen Handschrift $\mathrm{g}^{1}$ mit den Varianten der übrigen Zeugen abgedruckt: Der deutsche Facetus. Berlin 1911 (Palaestra 86), S. 221-241.

${ }^{4}$ Vgl. zusammenfassend zu Cato und Facetus im lateinischen Trivialunterricht und zu ihren Übersetzungen ins Deutsche Nikolaus Henkel: Deutsche Übersetzungen lateinischer Schultexte. Ihre Verbreitung und Funktion im Mittelalter und in der frühen Neuzeit. Mit einem Verzeichnis der Texte. München 1988 (MTU 90), S. 228-231 bzw. S. 245-248. Speziell zu den lateinisch-deutschen Cato-Facetus-Verbindungen habe ich an anderer Stelle eine vollständige Belegübersicht gegeben: Textreiben in der Mitüberlieferung von Schultexten als Verschriftlichungsphänomen. Typen ibrer Herausbildung im Lateinischen ('Liber Catonianus', 'Auctores octo') und in der Volkssprache ('Cato'/ 'Facetus'). In: Rudolf Suntrup, Jan Veenstra (Hrsg.): Erziehung, Bildung, Bildungsinstitutionen im Spätmittelalter und Früher Neuzeit. Frankfurt/M. [u.a] (Medieval to early modern culture / Kultureller Wandel vom Mittelalter zur Frühen Neuzeit 6) [im Druck]. 
1. Cato

Präzisere Konturen lassen sich der Cato- und Facetus-Überlieferung im Hausbuch verleihen, wenn man für beide Werke in deren weiterreichende Text- und Überlieferungsgeschichte ausholt. Was zunächst den Cato betrifft, lässt sich allein mit den Angaben des Hausbuch-Registers nicht bestimmen, welche der zahlreichen Übersetzungsfassungen den lateinischen Text einst begleitet hat, wohl aber von der Überlieferungsgeschichte her einiges, wenn auch teils nur mit mehr oder minder großer Wahrscheinlichkeit, ausschlieBen und anderes nahelegen. Bezieht man hier zunächst jene Textzeugen in die Betrachtung ein, die deutsche Übersetzungen der 'Disticha Catonis' entweder noch vor der oder doch zumindest zeitnah zur Entstehung des Hausbuchs belegen, dann kommen um 1350 überhaupt nur zwei von ihnen als Begleitung des lateinischen Textes in Frage: 1. der nach der Herkunft seiner ältesten, bereits im frühen 14. Jahrhundert niedergeschriebenen Handschrift aus dem Zisterzienserstift Zwettl benannte 'Zwettler Cato'; ${ }^{5}$ 2. die sogenannte Rumpfbearbeitung, also die einzige seinerzeit von Friedrich Zarncke in seiner Untersuchung zum deutschen Cato im Text vollständig hergestellte Übersetzungsfassung (und die deshalb dem Mittelaltergermanisten am ehesten als "deutscher Cato" vertraute), die vor der Mitte des 14. Jahrhunderts bereits die Benediktbeurer Bruchstücke, ${ }^{6}$ die einst Kaloczaer Handschrift ${ }^{7}$ und ihre Schwesterhandschrift des Heidelberger Cpg $341,{ }^{8}$ die Melker Stricker-Sammlung ${ }^{9}$ und die des Reg. lat. $1423^{10}$ sowie der Cgm $717^{11}$

${ }^{5}$ Zwettl, StiB, Cod. 357, 89ra-98vb; vgl. Charlotte Ziegler: Zisterzienserstift Zwettl. Katalog der Handschriften des Mittelalters. Bd. 4: Codex 301-424. Zwettl 1996/97 (Scriptorium Ordinis Cisterciensium Monasterii B. V. M. in Zwettl 4), S. 193-196.

${ }^{6}$ München, BSB, Cgm 5249/29b; vgl. Karin Schneider: Die Fragmente mittelalterlicher deutscher Versdichtung der Bayerischen Staatsbibliothek München (Cgm 5249/179). Stuttgart 1996 (ZfdA, Beiheft 1), S. 59f.; Hartmann von Aue. Der arme Heinrich, hrsg. von Hermann Paul. 16. Aufl. besorgt von Kurt Gärtner. Tübingen 1996 (ATB 3), S. XV-XVIII.

${ }^{7}$ Heute aufbewahrt in Genf-Cologny, Bibliotheca Bodmeriana, Cod. Bodmer 72; vgl. René Wetzel: Deutsche Handschriften des Mittelalters in der Bodmeriana. Mit einem Beitrag von Karin Schneider zum ehemaligen Kalocsa-Codex. Cologny-Genève 1994 (Bibliotheca Bodmeriana. Kataloge 7), S. 81-129 und Abb. 6-8.

${ }^{8}$ Wetzel (Anm. 7), S. 81-84, S. 129.

${ }^{9}$ Melk, StiB, Cod. 1547 (1859, 1848, R 18); vgl. Christine Glassner: Inventar der Handschriften des Benediktinerstiftes Melk. Teil 1: Von den Anfängen bis ca. 1400. Katalogband. Wien 2000 (Österreichische Akademie der Wissenschaften. Phil.-hist. Kl. Denkschriften 285; Veröffentlichungen der Kommission für Schrift- und Buchwesen des Mittelalters. Reihe II, Bd. 8, Teil 1), S. 422-425; Die Kleindichtung des Strickers, hrsg. von Wolfgang Wilfried Moelleken. Göppingen 1973-78 (GAG 107), Bd. 1, S. XXXI.

10 Vgl. Carl Greith: Spicilegium Vaticanum. Beiträge zur näheren Kenntnis der Vatikanischen Bibliothek für deutsche Poesie des Mittelalters. Frauenfeld 1838, S. 57-66; Friedrich Zarncke: Geschichte der deutschen Übersetzungen der im Mittelalter unter dem Namen Cato bekannten Distichen bis zur Verdrängung derselben durch die Übersetzung Seb. Brants am Ende des 15. Jahrh. Leipzig 1852. Nachdr. Osnabrück 1966, S. 12f. und S. 188; Moelleken (Anm. 9), Bd. 1, S. XXXII.

${ }^{11}$ München, BSB, Cgm 717; vgl. Das Münchner Gedicht von den 15 Zeichen vor dem Jüngsten Gericht. Nach der Handschrift der Bayerischen Staatsbibliothek Cgm 717. Edition und Kommentar, hrsg. von Christoph Gerhardt und Nigel F. Palmer. Berlin 2002 (Texte des späten Mittelalters 41). 
tradieren. ${ }^{12}$ Von den zwar erst später überlieferten, aber unabhängig davon vor dem oder zeitnah zum Hausbuch datierenden Übersetzungen sind hingegen keine zum Vergleich in Betracht zu ziehen. Stephans von Dorpat um die Mitte des 14. Jahrhunderts verfertigte Cato-Übertragung fällt schon wegen des niederdeutschen Sprachstandes und überdies auch deshalb für das Hausbuch aus, weil sich auch ihre Verbreitung ganz auf den niederdeutschen Sprachraum beschränkt. ${ }^{13}$ Vergleichbares gilt für den vor 1350 verfassten 'Niederrheinischen Cato' mittelfränkischer Sprachprägung und seine westmitteldeutschen/ westniederdeutschen Handschriften. ${ }^{14}$

Natürlich kann eine selbstständige Übersetzung, die vielleicht nur vom Hausbuch weitergegeben wurde, ebensowenig mit letzter Sicherheit ausgeschlossen werden wie mit der Möglichkeit zu rechnen ist, dass einige der Cato-Übersetzungen mit später einsetzender Überlieferung noch ins zeitliche Umfeld des Hausbuchs gehören und damit als potentielle Ergänzung des lateinischen Textes in Frage kommen. ${ }^{15}$ Hält man sich indes an das Gegebene, müssen zuerst der 'Zwettler Cato' und mehr noch die Rumpfbearbeitung in Betracht gezogen werden. "Mehr noch" muss die Rumpfbearbeitung in Betracht gezogen werden sowohl wegen ihrer bedeutend breiteren Bezeugung schon vor der Entstehung des Hausbuchs als auch, weil der 'Zwettler Cato' eine Textradition vertritt, die über die genannte älteste Zwettler Handschrift hinaus lediglich noch in zwei weiteren Handschriften zu greifen ist. Dagegen sind von der Rumpfbearbeitung neben den genannten noch über 30 weitere Textzeugen bekannt, und sie fand schließlich sogar noch Eingang in den Buchdruck. ${ }^{16}$

12 Nur pauschal ins 14. Jahrhundert datieren lässt sich die 1809 in der Regensburger Jesuitenbibliothek aufbewahrte, verbrannte Handschrift, die Andreas Hirn bekannt gemacht hat: Katho des Maysters Rat. Idunna und Hermode 1812, Nr. 14, S. 53-55 und Nr. 15, S. 57-59; vgl. Zarncke (Anm. 10), S. 16 und S. 188.

${ }^{13}$ Datierung nach Hartmut Beckers: Stephan von Dorpat. In: ${ }^{2}$ VL 9 (1995), Sp. $290-$ 293, hier Sp. 290; ebd. auch eine Übersicht über die Textzeugen, die sich noch um das Bruchstück Berlin, SBB-PK, Mgq 643 ergänzen lässt.

${ }^{14}$ Datierung nach Peter Kesting: Cato. In: ${ }^{2}$ VL 1 (1978), Sp. 1192-1196, hier Sp. 1194f.; Textzeugen bei Zarncke (Anm. 10), S. 160 (Berlin, SBB-PK, Mgf 579) und S. 170 (ehemals Kloss Nr. 4559, inzwischen Berlin, SBB-PK, Mgf 1060); Wilhelm Grimm: Vridankes Bescheidenheit. Göttingen 1834, S. VIII (Kassel, LBuMB, $8^{\circ}$ Ms. philos. 5); Paul Graffunder: Cato's Distichen in niederrheinischer Übersetzung. Programm des Königlichen Prinz Heinrichs-Gymnasiums 1896/97. Berlin 1897, S. 4-6; J. Franck: Aus dem historischen Archiv der Stadt Köln. ZfdA 44 (1900), S. 117-131, hier S. 199-123 (inzwischen Köln, HA, Best. 7020 Nr. 320); Henkel (Anm. 4), S. 230 (Frankfurt, StuUB, Fragm. germ. III 4 - bei Henkel noch ohne Textzuordnung nachgetragen).

${ }_{15}$ Noch im 14. Jahrhundert setzt beispielsweise die Überlieferung des von Zarncke so benannte, nur in Textproben zugängliche Cato "in zwielichtem dialecte" (vgl. Zarncke [Anm. 10], S. 162-170) und der Zarncke noch unbekannte schlesische Cato (vgl. Zatoćil [Anm. 2], S. 183-229) ein.

${ }_{16}$ Die vollständigste Zusammenstellung bietet nach wie vor Zarnckes Untersuchung, die bereits 26 - richtiger 25, denn die von ihm unter der Sigle h geführte ist identisch mit seiner Sigle k - Textzeugen aufführt. Ergänzungen bieten v.a. Dieter Harmening: Neue Beiträge zum deutschen Cato. ZfdPh 89 (1970), S. 346-368, und Henkel (Anm. 4), S. 230. Eine vollständige Übersicht bietet das über http://www.rrz.uni-hamburg.de/distichacatonis/ zu erreichende Internet-Verzeichnis 'Disticha Catonis' - Datenbank der deutschen Übersetzungen. 
Lediglich ein weiterer überlieferungsgeschichtlicher Befund könnte in diesem $\mathrm{Zu}$ sammenhang dann noch irritieren. Im Unterschied zum 'Zwettler Cato', den in der Zwettler Handschrift sein lateinisches Pendant begleitet, kennzeichnet die Rumpfbearbeitung eine durchweg einsprachige Tradierung. Diese Übersetzungsfassung wurde im 13., 14. und 15. Jahrhundert ausnahmslos ohne den lateinischen Text tradiert. ${ }^{17}$ Das passt zunächst gar nicht zum zweisprachigen Cato des Hausbuch-Registers. Allerdings führt der im Hausbuch anschließende Facetus in zunächst ähnlich widersprüchliche Verhältnisse.

\section{Facetus}

Das inzwischen über den Ausstellungskatalog von Brunner und Schmidt leicht in Abbildung zugängliche Münchner Facetus-Fragment zeigt jene zweispaltige und 32-zeilige Seiteneinrichtung, die auch den zweiten Band des Hausbuchs über weite Strecken prägt. ${ }^{18} \mathrm{Da}$ das erhaltene Blatt jedoch der Länge nach halbiert wurde, ist nur noch eine Textspalte zu sehen, und zwar, wie aus dem Textanschluss hervorgeht, die äußere: Auf der äußeren Spalte der recto-Seite stehen V.31-62 des Facetus der Übersetzungsfassung $\mathrm{G}\left(=\mathrm{G}, \mathrm{g}^{1}, \mathrm{~g}^{2}\right)$, die in der äußeren Spalte der verso-Seite mit V.63-94 ohne Lücke - das ist an den Parallelhandschriften zu überprüfen - fortgesetzt werden. Von lateinischem Text, den das Register führt, ist auf dem Bruchstück nichts zu erkennen. Dass er in der Innenspalte gestanden hätte, lässt sich angesichts der oberhalb des Schriftspiegels in der am Blattrand noch in Resten erkennbaren, in eigener Farbe ausgeführten Überschrift $<\ldots>o c h$ / gemeit ausschließen, die Ehrismann überzeugend zu Das buoch gemeit zu ergänzen und als in Entsprechung zu Liber Facetus gebildete Textüberschrift zu lesen vorschlägt. ${ }^{19} \mathrm{Er}$ kann nur vorangegangen oder, zumindest theoretisch, der Übersetzung gefolgt sein, wobei freilich eine Nachstellung des lateinischen Textes ganz ungewöhnlich wäre. Wahrscheinlicher ist, dass das Register ganz richtig, nämlich sehr präzise von einem facetus Cum nichil utilius [zuerst] zu latin vnd [dann anschließend] in tütsch berichtet.

Beachtenswert ist weiterhin, dass eine Übersetzungsfassung ins Hausbuch übernommen wurde, der allein hier der lateinische Text mitgegeben ist, die hingegen andernorts überhaupt nur in durchweg einsprachige Textgemeinschaften übernommen wurde. Sowohl in $\mathrm{g}^{1}$ (Forschungsbibliothek Gotha, Chart. A 216) ${ }^{20}$ wie in der 'Liedersaal-Hand-

17 Henkel (Anm. 4), S. 178.

18 Brunner/Schmidt (Anm. 3), S. 35 (leider mit vertauschter Vorder- und Rückansicht, denn $\mathrm{Bl} .5 \mathrm{r}$ ist rechts, $\mathrm{Bl} .5 \mathrm{v}$ links abgebildet).

19 Ehrismann (Anm. 3), S. $284 \mathrm{f}$.

${ }^{20}$ Friedrich Jacobs, Friedrich August Ukert (Hrsg.): Beiträge zur ältern Litteratur oder Merkwürdigkeiten der herzoglichen öffentlichen Bibliothek zu Gotha. Leipzig 1835-38, Bd. 2, S. 294-300; Schröder (Anm. 3), S. 221 (“ist vielleicht Abschrift von G”); Tilo Brandis: Mittelhochdeutsche, mittelniederdeutsche und mittelniederländische Minnreden. Verzeichnis der Handschriften und Drucke. München 1968 (MTU 25), S. 230 (“Bl 75-111: Würzburg um 1400"); Hanns Fischer: Studien zur deutschen Märendichtung. 2., durchgesehene und erweiterte Auflage besorgt von Johannes Janota. Tübingen 1983, S. 201f. (der entsprechende Faszikel der Handschrift um 1400 geschrieben und Würzburger Provenienz), S. 288; Gerd Dicke, Klaus Grubmüller: Die Fabeln des Mittelalters und der frühen Neuzeit. Ein Katalog der deutschen Versionen und ibrer lateinischen Entsprechungen. München 1987 (Münstersche Mittelalter-Schriften 60), S. 350 (K 301); Brunner/Schmidt (Anm. 3), S. 52f. ("Corpus von 18 deutschen Reimpaargedichten, die vielleicht [...] in Würzburg entstanden sind”). Vgl. Korrekturnachtrag Anm. 37. 
schrift' $\mathrm{g}^{2}$ (Karlsruhe, BLB, Cod. Donaueschingen 104) ${ }^{21}$ begleitet den deutschen Facetus v.a. kleinere Reimpaardichtung (Fabeln, Bîspel, Mären, Minnereden). Überlieferungstypologisch stellt sich diese Übersetzungsfassung damit genau zur Rumpfbearbeitung des Cato. Hier wie dort handelt es sich um Übertragungen, die zuerst den einsprachigen Laien anvisieren, nicht aber den zweisprachigen Lateinschüler. Zufällig enthält die ehemals Donaueschinger Handschrift neben dem deutschen Facetus auch noch einen deutschen Cato. Keineswegs zufällig handelt es sich bei diesem eben um eine Rumpfbearbeitung.

Die oben angedeuteten Bedenken gegen eine Verbindung der 'Disticha Catonis' mit der Rumpfbearbeitung statt mit dem 'Zwettler Cato' sind also vom Facetus her zu entkräften. Zu beachten ist dann nur, dass sich der lateinische und der deutsche Text der Rumpfbearbeitung nicht in der Art der für den Lateinunterricht angefertigten Übertragungen eins zu eins entsprechen. Bekanntlich ordnet die Rumpfbearbeitung ihre Verse gegen die lateinischen Vorlage, stellt, von inhaltlichen Erwägungen geleitet, Verse um, vernachlässigt Verse der Vorlage und ergänzt gegen sie. Für eine Parallelführung des lateinischen und deutschen Textes in zwei Spalten oder gar für eine Einschaltung der deutschen Reimpaarverse in den fortlaufenden Verstext der lateinischen Vorlage fehlen damit textseitig die Voraussetzungen. Wenn es die Rumpfbearbeitung war, die die 'Disticha Catonis' im Hausbuch begleitete, dann ließ sich also darbietungstechnisch einzig eine Nachstellung des deutschen Textes hinter den lateinischen realisieren. Eben diese Nachstellung wurde aber bereits vom Facetus-Fragment nahegelegt. Auch für den Cato ist also davon auszugehen, dass das Register die Tatbestände sehr präzise benennt: [zuerst] DEr katho zu latin. vnd [anschließend] zú tútsche.

\section{Zum Nach-, Neben- und Ineinander von Latein und Deutsch im 14. Jahrhundert}

Die in ihrem Layout demnach höchstwahrscheinlich einheitlich gestaltete CatoFacetus-Partie, in der auf den vorangestellten lateinischen Text jeweils separat der deutsche folgte, ist Teil des zwischen 1345 und 1347 begonnenen Grundstocks des Hausbuchs. ${ }^{22}$ Sie steht in keiner engeren Verbindung zu den sie unmittelbar umgebenden Texten, die nicht mehr auf die lateinische Schulpraxis verweisen. ${ }^{23}$ Die lateinische Schulpraxis hat allenfalls die Anregung zur Zusammenstellung der beiden lateinischen Texte vermittelt, ${ }^{24}$ darf jedoch für ihre Übersetzungen ins Deutsche nicht mehr unmittelbar in Anspruch genommen werden. Die Rumpfbearbeitung, die wohl ohne größere Suche zur Hand war, entstammt außerschulischen, laikalen Gebrauchszusammenhängen, der deutsche Facetus in der vorliegenden Fassung vermutlich nur einer uns in ihren genauen

${ }^{21}$ Zusammenfassend zur Handschrift Klaus Grubmüller: 'Liedersaal-Handschrift'. In: ${ }^{2}$ VL 5 (1985), Sp. 818-822 (mit weiterer Literatur); vgl. zum neuen Aufbewahrungsort Felix Heinzer: Die neuen Standorte der ehemals Donaueschinger Handschriftensammlung. Scriptorium 49 (1995), S. 312-319.

${ }_{22}$ Vgl. Kornrumpf/Völker (Anm. 1), besonders S. 69.

${ }^{23}$ Nämlich DE remedijs salutiferis et laudabilibus ordinacionibus Magistri Mychaelis prefati. pro eius et suorum successu prospero et salubri in hoc seculo et futuro und Die funfzig gruz vnser frauwen; vgl. zur Identifizierung jeweils Kornrumpf/Völker(Anm. 1), S. $74 f$.

${ }^{24}$ Mit Keyser (Anm. 1), S. 37-44 und S. 166-169 lässt sich ein Rückgriff auf Vorlagen vermuten, die in der Bibliothek des Neumünsterstifts bewahrt wurden. 
Umrissen nicht mehr sichtbaren Würzburger Lokaltradition - wenn er nicht gar erst für das Hausbuch übersetzt wurde, das den ältesten Textzeugen stellt und aus dem mit $\mathrm{g}^{1}$ noch ein halbes Jahrhundert später, wiederum in Würzburg, eine Abschrift genommen wurde. $^{25}$

Die Entscheidung für eine zweisprachige Darbietung zweier verbreiteter Unterrichtstexte schließlich stellt eine vom Ansinnen, ein "Lehr- und Lese-Buch für künftige Generationen" ${ }^{26}$ zusammenzustellen, angestoßene Eigenleistung des Michael de Leone dar. Denn damit betrat der Würzburger Protonotar Neuland. Die ersten Handschriften mit zweisprachiger Darbietung des 'Facetus Cum nihil utilius' entstehen erst in der zweiten Hälfte des 14. Jahrhunderts (Jena, UuLB, Ms. Prov. o. 17; Frankfurt, StuUB, Fragm. germ. III, 2rv). ${ }^{27}$ Beim Cato ließ sich die bis zum Hausbuch einzig einsprachig verbreitete Rumpfbearbeitung nicht als Vorbild nehmen. Der einzige E noch vorangehende zweisprachige Cato aus Zwettl hingegen führt in eine Handschrift, deren weiterer Inhalt der Schule durchaus fernsteht und keineswegs eingespielte Traditionen erkennen lässt, wie schriftliche Unterrichtsmaterialien ihren Lesern zweisprachig darzubieten gewesen wären. Im Gegenteil beschritt man im 'Zwettler Cato' sogar einen Weg, der sich später gerade nicht durchsetzte: Die deutsche Gesamtübersetzung und der lateinische Text laufen in zwei parallelen Spalten nebeneinander her. ${ }^{28}$ Leones lateinisch-deutscher Textverbund führt also noch in eine Phase des Experimentierens mit verschiedenen Darbietungsformen des Lateinischen und Deutschen hinein. Er ist eben deshalb - sozusagen als "positiver" Beleg - überaus bedeutsam, weil gerade im Bereich der Überlieferung von Schultexten überall dort, wo zeitliche Vorgänge der Durchsetzung von Phänomen erfasst werden sollen, wegen der hohen Verlustquote von Handschriften prinzipiell schlecht mit Nicht-Nachweisbarkeiten, die ja immer einfach auch auf Verlust beruhen können, argumentiert werden kann. ${ }^{29}$

Experimente glücken oder gehen schief. Den Protonotar wird es nicht gekümmert haben, dass sein Versuch zweisprachiger Textdarbietung elementarer Unterrichtstexte keine Nachfolge fand, denn er entstand im Rahmen auf das eigene Geschlecht gerichteter Hausüberlieferung, ${ }^{30}$ nicht für den institutionalisierten Lateinunterricht. Dieser geht

${ }^{25} \mathrm{Vgl}$. oben Anm. 20.

${ }^{26}$ Kornrumpf (Anm. 1), Sp. 499. Vgl. jetzt auch Christa Bertelsmeier-Kierst: Das Hausbuch des Michael de Leone. Zu Programm und Struktur der Sammlung. In: Horst Brunner (Hrsg.): Würzburg, der Große Löwenhof und die deutsche Literatur des Spätmittelalters. Wiesbaden 2004 (Imagines medii aevi 17), S. 199-210. Ich danke Frau Bertelsmeier-Kierst dafür, dass sie mir ihren Beitrag noch vor der Drucklegung zugänglich gemacht hat.

${ }_{27}$ Vgl. Franzjosef Pensel: Verzeichnis der altdeutschen Handschriften und ausgewählter neuerer deutscher Handschriften in der Universitätsbibliothek Jena. Berlin 1986 (DTM 70; Verzeichnis altdeutscher Handschriften in der Deutschen Demokratischen Republik 2), S. 417 f., bzw. Gerhardt Powitz: Mittelalterliche Handschriftenfragmente der Stadt- und Universitätsbibliothek Frankfurt am Main. Frankfurt/M. 1994 (Kataloge der Stadt- und Universitätsbibliothek Frankfurt am Main 10,6), S. 155.

${ }^{28} \mathrm{Vgl}$. Henkel (Anm. 4), S. 86f. (mit Abbildung von 94r) und S. 156f. Anm. 11.

${ }^{29}$ Vgl. grundsätzlich Arnold Esch: Überlieferungschance und Überlieferungszufall als methodisches Problem des Historikers. Historische Zeitschrift 240 (1985), S. 529-570.

${ }^{30}$ Einschlägig ist eine dem Register vorangestellte Vorbemerkung, die den Doppelband iedem von sinem geslecht. der danne den selben hoff [sc. den Löwenhof, M.B.] innehat (1va) überantwortet. 
schon deshalb einen anderen Weg, weil die Rumpfbearbeitung dort der vollständigen Erschließung der lateinischen Vorlage nicht dienen konnte. Überdies erscheint es für den Unterricht unpraktischer, den deutschen dem lateinischen Text in Gänze nachzuordnen als die deutschen Reimpaare auf der handschriftlichen Seite jeweils dort zu platzieren, wo sie der Erschließung ihrer lateinischen Gegenüber unmittelbar dienen, also jeweils im unmittelbaren Anschluss an die lateinischen Verse. (Auch das Zwettler Modell erscheint in dieser Hinsicht, weil das Auge zwischen den Spalten hin- und herspringen muss, noch unausgereift.) Im Lateinunterricht wird sich vielmehr die alternierende Darbietung durchsetzen, in der lateinischer und deutscher Text sich jeweils über kurze Strecken abwechseln - und dies zudem erst in den Jahrzehnten nach der Anlage des Hausbuchs. Die zwei ältesten entsprechend zweisprachig angelegten Facetus-Handschriften wurden oben genannt; für den zweisprachigen Cato sind aus der Zeit vor 1400 neben dem oben angeführten Frankfurter Bruchstück (das dem Facetus einen 'Niederrheinischen Cato' voranstellt) aus der Zeit vor 1400 nur noch London, BL, MS Additional $11250,12 \mathrm{r}-13 \mathrm{v},{ }^{31}$ und das vielleicht noch ins 14. Jahrhundert zu setzende, heute vermisste Znaimer Fragment ${ }^{32}$ sowie mehrere Textzeugen des 'schlesischen Cato' zu nennen, ${ }^{33}$ die Überprüfung an einem weiteren noch im 14. Jahrhundert in Unterrichtshandschriften zu belegenden zweisprachigen Ensemble, am 'Distigium'/'Cornutus' des Johannes de Garlandia, ${ }^{34}$ bestätigt das Bild: Die alternierende Darbietung lateinischen und deutschen Textes setzt sich in den schriftlichen Unterrichtsmaterialien erst in der zweiten Jahrhunderthälfte durch..$^{35}$

Man muss sich bewusst halten, dass die Verzahnung des deutschen mit dem lateinischen Text nicht weniger erforderte als den lateinischen Ausgangstext gleichsam "aufzusprengen”. Dafür brauchte es eines drängenden praktischen Anstoßes, eines breiter einsetzenden Verlangens nach systematisch und auch in schriftlicher Form zugänglichen Erschließungshilfen des Textstudiums in der Volkssprache. Im 14. Jahrhundert schlägt sich dieses Verlangen in zahlreichen neuen Übersetzungen von Unterrichtstexten ins Deutsche nieder, aber auch im Aufstieg der Volkssprache zum statt sporadisch nun systematisch für eine schriftliche Wort-für-Wort-expositio genutzten Erschließungsin-

31 Vgl. Robert Priebsch: Deutsche Handschriften in englischen Bibliotheken. Erlangen 1896-1901. Nachdr. Hildesheim/New York 1976, Bd. 2, S. $107 f$.

${ }^{32}$ Vgl. Julius Feifalik: Cato. Notizenblatt der historisch-statistischen Section der Kaiserlich-Königlichen Mährischen Gesellschaft zur Beförderung des Ackerbaues, der Natur- und Landeskunde 1 (1855), S. 59f. (mit Textabdruck).

${ }_{33}^{33}$ Vgl. die Belegübersicht in meinem in Anm. 4 genannten Beitrag.

${ }^{34}$ Vgl. Der Deutsche Cornutus. I. Teil. Der Cornutus des Johannes de Garlandia, ein Schulbuch des 13. Jahrhunderts. In den deutschen Übersetzungen des Mittelalters zum ersten Male hg. von Edwin Habel. Berlin 1908, S. 8f.; Franz Josef Worstbrock: Johannes de Garlandia. In: ${ }^{2}$ VL 4 (1983), Sp. 612-623, hier Sp. $618 f$.

${ }^{35}$ Henkel (Anm. 4), vgl. besonders S. 122-131 und S. 156f., geht dem Darbietungstyp nicht weiter nach; Palmer klammert ihn aus seiner instruktiven Übersicht leider aus: Nigel F. Palmer: Zum Nebeneinander von Volkssprache und Latein in spätmittelalterlichen Texten. In: Ludger Grenzmann, Karl Stackmann (Hrsg.): Literatur und Laienbildung im Spätmittelalter und in der Reformationszeit. Symposion Wolfenbüttel 1981 (Germanistische Symposien. Berichtsbände 5), S. 579-600 [Diskussionsbericht von Joachim Behr S. 601-603] - vgl. hier besonders S. 592f. 
strument oder in der Herausbildung zweisprachiger Erstlesebücher. ${ }^{36}$ Die nur auf das eigene geslecht gerichteten Intentionen des Würzburger Protonotar Michael de Leone hingegen erscheinen gegenüber solchen Anstößen als ein zu schwacher Motor für Innovationen größerer Reichweite. ${ }^{37}$

Wuppertal

Michael BALDZuHN

${ }^{36}$ Dass die sogenannten "Wort-für-Wort-Übersetzungen" lediglich die als Bestandteil des lateinischen Kommentars bekannte expositio ad litteram in die Volkssprache überführen, deren Erläuterungen damit freilich im 14. Jahrhundert einen neuen Status erhalten, dafür habe ich an anderer Stelle argumentiert: 'dem selbigen glosiert er allwegen in die feder'. Distribution und Produktion/Rezeption volkssprachlicher 'expositio ad litteram' im Umfeld des spätmittelalterlichen Trivialunterrichts. In: Elizabeth Andersen, Manfred Eikelmann, Anne Simon (Hrsg.): Texttyp und Textproduktion in der deutschen Literatur des Mittelalters. Kolloquium Newcastle 2001. Berlin 2005; zu Aufkommen und Verbreitung zweisprachiger Erstlesebücher im 14. Jahrhundert verweise ich auf den in Anm. 4 genannten Beitrag.

${ }^{37}$ Korrekturnachtrag: Die vorgetragenen Befunde erhalten durch die jüngst vorgeschlagene Vordatierung des Textzeugen $\mathrm{g}^{1}$ des 'Facetus Cum nibil utilius' (vgl. Falk Eisermann: Zur Datierung der 'Würzburger Kleinepiksammlung' [Forschungsbibliothek Gotha, Chart. A 216]. ZfdA 134 [2005], S. 193-204), der statt "um 1400" offenbar noch zu Lebzeiten Michaels de Leone niedergeschrieben wurde (1342-45), ein noch schärferes Profil: Die zweisprachige Darbietung des Facetus im Leone-Hausbuch tritt vor diesem Hintergrund noch deutlicher als singuläres Experiment hervor. 